CENTRO DE ESTUDIOS DE DERECHO PENAL. "Revista de Revistas."

Polit. crim. No 8, 2009, R1-8, pp. 1-13.

[http://www.politicacriminal.cl/n_8/r_1_8.pdf]

\title{
Recensión: Rivista Trimestrale di Diritto Penale dell'Economia, año XXI, n 3 y 4, 2008
}

\author{
Raúl Carnevali R. \\ Doctor en Derecho. Profesor Asociado de Derecho Penal de la Universidad de Talca y \\ Subdirector de su Centro de Estudios de Derecho Penal \\ rcarnevali@utalca.cl
}

En el volumen 3 de la revista se encuentran los siguientes trabajos en la sección doctrina: Guido Casaroli, de la Universidad de Ferrara, con el trabajo titulado "Acerca de los criterios de imputación de la responsabilidad por el delito a la persona jurídica" (pp. 561602), examina los alcances del decreto legislativo 231 de 2001 por el cual se reconoce normativamente la criminalidad colectiva de las empresas, estableciendo así el principio societas puniri debet. Se aborda también, si la naturaleza real de la responsabilidad de las personas jurídicas es de carácter administrativo o penal, considerando el autor que se trataría de una responsabilidad de orden penal. Más adelante, desde la perspectiva de la atribución de responsabilidad se examina si se está frente a una verdadera culpabilidad de empresa o en cambio, a una culpabilidad de la persona física autora de la infracción, que afecta a la persona jurídica llamada a responder. Pues bien, una vez reafirmada la distinción entre el hecho cometido por los sujetos que ocupan una posición dominante y los realizados por los subalternos, es posible extraer un criterio particular de imputación fundado en una culpabilidad de organización, que es autónoma y que se conforma por un déficit en la adopción de medidas preventivas, traducidas en modelos de organización y gestión adecuadas. Conforme a lo anterior, respecto a la creación de una incriminación autónoma para fines de atribución de responsabilidad, el legislador ha introducido una eximente para aquellos casos en donde la infracción ha sido cometida por los directores, probándose que se han adoptado las medidas preventivas conforme a un modelo de organización y de gestión. También el autor examina aquellos casos en donde la infracción es cometida por los subalternos. Sigue la sección doctrina con el trabajo de Abraham Castro Moreno, de la Universidad Carlos III de Madrid, titulado "Los delitos contra la propiedad industrial a la luz de la jurisprudencia española" (pp. 603-668). El texto se encuentra también publicado en idioma español. Se analiza la legislación penal española en materia de competencia, en donde se encuentran los delitos contra la propiedad industrial, comprendiéndose, entre otras, la conductas que atentan a las patentes o modelos de utilidad; patentes secretas; marcas o signos distintivos y denominaciones de origen. Se destaca de este trabajo en que no sólo se estudian los elementos teóricos, sino que también su aplicación práctica, examinando la jurisprudencia. Continúa la sección con el artículo de Pasquale Fimiani, juez de la Corte de Casación, titulado "Residuos y subproductos: viejo y nuevo límite en el Derecho penal del ambiente" (pp. 669-702). El autor aborda el decreto legislativo 152 de 2006 denominado Texto Único del medio ambiente, por el cual se introdujo la noción de subproducto, siguiendo de este modo la decisión de la Corte de Justicia de las Comunidades Europeas. En efecto, ésta había previsto la noción de subproducto, aunque delimitado a la reutilización y sin transformación preliminar de un bien, de un material o de una materia prima en el circuito de producción. Para el autor surgen ciertos espacios de incertidumbre en cuanto a saber si es o no apropiado hablar de subproductos y no de desechos, respecto de 
CARNEVALI, Raúl, "Recensión: Rivista Trimestrale di Diritto Penale dell'Economia, año XXI (2008), fascículos 1 y 2" Polít. crim. No 8, 2009, R1-8, pp. 1-3.

ciertos tratamientos como la limpieza o selección de bienes. A continuación, Paolo Patrono, en su trabajo titulado "La regulación penal de la contaminación hídrica y atmosférica después del llamado Texto Único ambiental: nuevos y viejos aspectos problemáticos" (pp. 703-723), analiza la protección de medio ambiente en lo que concierne a la lucha contra polución hídrica y atmosférica después de la aprobación de decreto legislativo 152 de 2006, llamado Texto Único del medio ambiente, posteriormente modificado y completado por el decreto legislativo 4 de 2008. El autor afirma que el Derecho penal del medio ambiente depende del Derecho administrativo. Así se observa a través de las contravenciones, infracciones formales, delitos de desobediencia y de peligros presuntos. Asimismo, señala la importancia de incorporar las infracciones al medio ambiente al Código penal, como también la necesidad de introducir, respecto de dichas infracciones, normas que rijan la responsabilidad de las personas jurídicas.

En la sección actualidad se encuentran los siguientes trabajos: "La autoridad para la vigilancia sobre los contratos públicos de trabajos, servicios y suministros: aspectos sancionatorios" de Luigi Giampaolino (pp. 739-751) y "La ley de ratificación y ejecución de la convención sobre cybercrime: consideraciones sustanciales y procesales" de Michele Guernelli (pp. 753-770).

En el volumen 4 de la revista se presentan tres artículos en la sección doctrina: Francesco Mucciarelli, de la Universidad L. Bocconi, con su trabajo "Los ilícitos de abuso de mercado, la responsabilidad del ente y la información" (pp. 823-861). La investigación examina las disposiciones en materia de abuso de mercado, centrándose en la noción de información, entendida como elemento que permite extraer plenamente el contenido del bien jurídico protegido. En esta materia es esencial el interés de los inversores de poder disponer de una información clara y correcta a fin de que puedan adoptar decisiones racionales sobre la base de un patrimonio informativo no viciado. Por tanto, señala el autor que es indispensable establecer procedimientos y controles capaces de identificar a través de esquemas de carácter general las posibilidades de riesgo y los actos que se deben prevenir. El siguiente trabajo es el de Licia Siracusa, "La competencia comunitaria en el ámbito penal al primer banco de prueba: la directiva europea sobre protección penal del ambiente" (pp. 863-902). La autora expone que la directiva 2008/99 CE sobre la protección penal del Medio Ambiente plantea uno de las primeras y más importantes pruebas de poder normativo comunitario a nivel penal. La norma tiene una fuerte importancia tanto desde una perspectiva política como jurídica. Política porque la Comisión y el Consejo deben resolver la admisibilidad de los instrumentos del Derecho comunitario clásico para los fines de aproximar las legislaciones nacionales penales. Jurídica, por cuanto la directiva entrega parámetros de referencia para la tipificación de las infracciones en materia medioambiental. En el trabajo de Luca Tumminello titulado "La mafia como método y como fin: la circunstancia agravante del art. 7 D.L. 152/1991, convertido en la L. 203/1991" (pp. 903945), examina el art. 7 del decreto ley 152 del 13 de mayo de 1991 convertido en ley 203 de 12 de julio de 1991 por el que se introdujeron dos circunstancias agravantes con efecto especial para la represión de la criminalidad organizada de carecer mafioso, a saber, la agravante del método mafioso y la agravante de la colusión mafiosa. La primera prevé una aumento de la pena para los delitos cometidos empelando las condiciones contempladas en el art. 416 bis del Código penal; la segunda agravante se refiere a aquellos actos cometidos a fin de favorecer la actividad de asociación de tipo mafioso. Se plantean importantes 
CENTRO DE ESTUDIOS DE DERECHO PENAL. "Revista de Revistas."

Polít. crim. No 8, 2009, R1-8, pp. 1-13.

[http://www.politicacriminal.cl/n_8/r_1_8.pdf]

problemas hermenéuticos vinculados a la identificación de sus elementos constitutivos y en relación a la compatibilidad o no con otras figuras criminales. Es así, que respecto al método se propone un análisis considerando los perfiles externos de la conducta criminal imputables a una metodología mafiosa: el contexto social de referencia. El autor también estudia otras circunstancias agravantes del art. 7, como también otras figuras.

En la sección actualidad se comprenden los trabajos de Paolo Bernasconi "Disposiciones coercitivas respecto a los bancos y empresas según el nuevo código suizo de procedimiento penal" (pp. 969-999); de Francesco Paolo Di Fresco "Mobbing y abuso funcionario" (pp. 1001-1013) y de Anna Silvia Scalco, " "Made in Italy»: necesidad de una más intensa tutela penal de la excelencia italiana en el mercado globalizado" (pp.1015-1034). 
MALDONADO, Francisco, "Recensión: Revista de Derecho Penal, no 26 y 27 (2009)" Polit. crim. No 8, 2009, R1-8, pp. 4-6.

\section{Recensión: Revista de Derecho Penal, $n^{0} 26$ y 27 (Enero y Mayo, 2009)}

Francisco Maldonado Fuentes

Doctor en Derecho. Profesor conferenciante Universidad de Talca y Director de extensión y programas especiales del Centro de Estudios de Derecho Penal

\section{fmaldonado@utalca.cl}

En el $\mathrm{n}^{\circ} 26$ la Revista incluye cuatro contribuciones en la Sección de "Artículos Doctrinales” (pp. 11-140). En la primera, de carácter más general, Silvia Barona Vilar aborda la "Mediación penal como pieza del sistema de tutela penal en el siglo XXI. Un paso mas hacia la resocialización y la justicia restaurativa" (pp. 11-53); El segundo y el tercero abordan objetos de estudio mas acotados, no por ello menos útiles pues suelen ser considerablemente más escasos. De ahí que sea útil tenerlos en cuenta para el evento en que debamos enfrentarnos a su análisis. Diaz-Maroto y Villarejo se dedica al tratamiento de "Las faltas contra os intereses generales en el Código Penal" (pp. 55-92) mientras que Fernández Hernández expone algunas "Consideraciones jurídico penales acerca de la piratería marítima" (pp. 93-119). La sección se cierra -como es habitual- con un aporte de derecho procesal que en esta oportunidad desarrolla Raquel Castillejo Manzanares exponiendo un trabajo sobre "La dispensa del deber de declarar del artículo 416 de la Ley de Enjuiciamiento Criminal respecto de la mujer que sufre violencia de género" (pp. 121140). La revista contiene además una amplia selección de "Jurisprudencia" (pp. 143-280), "Doctrina de la Fiscalía General del Estado" (pp. 283-296), una "Crónica Legislativa" (pp. 299-307) y un apartado de "Bibliografía” (pp. 311-314). Por su parte en el $n^{\circ} 27$ la Sección de "Artículos Doctrinales" contiene tres aportes de diversa naturaleza, uno de parte especial, otro de parte general y el tercero, como es habitual, dedicado a las cuestiones propias del derecho procesal (pp. 11-146). El primero, de Francisco Jiménez-Villarejo Fernández trata de "La delincuencia económica y las nuevas tecnologías: el fraude informático" (pp. 11-50), mientras que en el segundo Manuel Gallego Díaz ensaya una formulación novedosa acerca de "La aplicación de la pena en consideración a las circunstancias modificativas de la responsabilidad criminal: hacia una reordenación y simplificación de los distintos supuestos" (pp. 51-94). Finalmente, Ángeles G. Zarza se dedica a analizar "El principio de reconocimiento mutuo de las resoluciones judiciales en materia penal" (pp. 95-146). Se agrega como siempre una distendida selección de "Jurisprudencia" (pp. 149-314), una "Crónica Legislativa" (pp. 317-320), un apartado de "Bibliografía" (pp. 323-328) y una recensión de Begoña Vidal sobre el libro "Hacia un verdadero espacio judicial europeo" de L.M. Lujosa Vadell (pp. 329-331).

Quisiéramos en esta oportunidad dedicarle una mayor atención al aporte de la profesora Barona que se incluye en el $n^{\circ} 26$ de la revista, atendida la mayor amplitud de la temática que aborda y -sobre todo- su orientación práctica. En efecto y a diferencia de buena parte de los estudios que versan sobre medicación penal la autora no se limita a abordar las cuestiones habituales sino que, en base a los aspectos más asentados, procura realizar una apuesta de futuro acerca de la incorporación de la medicación penal en España, abordando asimismo los caracteres que se estima pueden regirla (pp. 41 y ss.). En efecto, constata la presencia de ventajas comparativas en el uso de esta institución como medio de resolución 
CENTRO DE ESTUDIOS DE DERECHO PENAL. "Revista de Revistas."

Polít. crim. No 8, 2009, R1-8, pp. 1-13.

[http://www.politicacriminal.cl/n_8/r_1_8.pdf]

de conflictos penales, a partir del sentido que tiene la recuperación del conflicto entre partes que configura cualquier caso penal. A partir de ello destaca el papel que juega la revitalización del rol de la víctima en el posicionamiento actual de la medicación (y en general, de las diversas modalidades de la llamada justicia restaurativa o conciliatoria) en atención a que minimiza el impacto de la (llamada) expropiación del conflicto por parte del Estado y, consecuencialmente el imperio de la legalidad como orientación estricta de la persecución penal (pp. 13-15; 18-22). Lo considera como una instancia evolutiva a partir de lo cual pronostica el que se trata de un proceso con efectos permanentes, minimizando los contenidos que permitirían calificarlo más bien como una especie de moda. Des taca en relación a ello el amplio reconocimiento que se le brinda a la mediación en los instrumentos internacionales, dedicando un espacio a su relación (pp. 15-18). Ahora bien, a la hora de describir los modelos evita toda disgregación, concentrándose, exclusivamente, en la forma como se prevé la relación entre las partes (presencia o secuencial) y en el momento procesal en que tiene lugar (antes, durante o después de que tenga lugar el proceso respectivo) siendo llamativo y novedoso el papel y utilidad que le asigna en sede de ejecución penal. En este último caso opera en las instancias de evolución de la reinserción que se muestran conflictivas, facilitando dicho proceso y la administración de la sanción (pp. 22-25). En materia de precedentes (que son descritos como verdaderos objetos de análisis, abordando fundamentalmente lo que considera son sus aciertos y desaciertos) menciona inicialmente los desarrollos llevados a cabo en el ámbito penal juvenil (pp. 2627), únicos que gozan de un directo reconocimiento de rango legal, como también las experiencias piloto propuestas e implementadas en torno a la regulación penal de adultos (pp. 28-30), acompañados del tratamiento referencia de algunas propuestas de derecho comparado (pp. 30 y ss.). Dedica especial atención al tratamiento que el ordenamiento alemán le concede a la mediación penal (pp. 31-40) lo que justifica en la mayor cercanía cultural y de sistemas jurídicos existente con el entorno español (p. 30). No hay que olvidar que en los modelos nórdicos tiene mayor presencia la discrecionalidad reglada y, sobre todo, una mayor disponibilidad de la persecución. En base a todo lo señalado describe las instancias donde estima que este recurso puede ser útil y posible de implementar (pp. 4142) y los elementos que deben caracterizar la mediación (pp. 42-54), incluyendo los aspectos que deben hacerse presentes en el mediador (pp. 48-50) y las posibles fases que lo configurarán (pp. 51-53). Desarrolla además lo que denomina es el ámbito objetivo de la mediación, donde ensaya los criterios a tener en cuenta para la selección del tipo de infracciones que pueden ser mediables (pp. 45-48). Quisiéramos destacar en esta oportunidad la presencia de algunas temáticas de relevancia que son abordadas en los extractos de sentencias contenidas en la sección de jurisprudencia en ambos números. En lo relativo al $\mathrm{n}^{\circ} 26$ quisiéramos en esta oportunidad destacar el extracto que se publica de la STS 503/2008, de 17 de Julio, que se ocupa profusamente del concepto de terrorismo e indaga acerca del compromiso que puede generar la presencia de delitos especiales fundados en él para la vigencia y respeto que debe darse al non bis in idem (pp. 183-196). La sentencia, por lo demás, versa sobre los atentados acaecidos en Madrid el 11 de Marzo de 2004, lo que permite atribuirle un significado especial. Con respecto al $n^{\circ} 27$ conviene apuntar que se incluye una sentencia del TC Español que resuelve cuestiones de inconstitucionalidad planteadas respecto de la Ley Orgánica de Medidas de protección Integral contra la violencia de género (STC 45/2009), que son en definitiva rechazadas (se 


\section{MALDONADO, Francisco, "Recensión: Revista de Derecho Penal,}

no 26 y 27 (2009)" Polit. crim. No 8, 2009, R1-8, pp. 4-6.

consideran extractos de los votos particulares disidentes) (pp. 166-180); Además, se considera una sentencia (657/2008) del TS Español que ratifica, como jurisprudencia sostenida, el que la muerte de un niño de pocos meses siempre es asesinato, obviando la exigencia subjetiva particular que tiene de exigir la doctrina nacional (chilena) para configurar la alevosía (pp. 196 a 200); otra (822/2008) del mismo órgano que acoge casos de tentativa relativamente inidónea (pp. 247-249), otra (891/2008) que aborda el problema de la causalidad en la producción de un resultado de muerte a través de un procedimiento médico cuando existen causas orgánicas en la víctima que la predisponen a dicho resultado (pp. 253-264); y, finalmente, otra (7/2009) sobre la valoración de la prueba de un coimputado cuando éste ya ha sido condenado por el mismo delito (pp. 273-277). 
CENTRO DE ESTUDIOS DE DERECHO PENAL. "Revista de Revistas."

Polít. crim. No 8, 2009, R1-8, pp. 1-13.

[http://www.politicacriminal.cl/n_8/r_1_8.pdf]

\title{
Recensión: Revista de Estudios de la Justicia, n $^{0} 11,2009$
}

\author{
Dr. Md. Reinaldo Bustos Domínguez \\ Coordinador Programa de Derecho y Bioética, Centro de Estudios de Derecho \\ Penal de la Universidad de Talca \\ rbustos@utalca.cl
}

El número 11 del año 2009 de la Revista de Estudios de la Justicia, el primero de tres en homenaje a la memoria del profesor Juan Bustos Ramírez, prolífico y brillante penalista y político recientemente fallecido, trae diez artículos doctrinarios y tres colaboraciones de amigos, discípulos y colegas que compartieron su interés académico.

El primero de ellos, "Un caso límite entre justificación y exculpación: la legítima defensa putativa" (pp. 13-34), del profesor español Francisco Muñoz C., donde analiza el par conceptual de los términos alemanes "Rechtfertigung" y "Entschuldigung" cuya traducción española es "justificación” y "exculpación”, como dos categorías analíticas útiles para una teoría general del derecho penal, aplicables en principio, a todas las culturas jurídicas, permitiendo ilustrar bastante bien la distinta naturaleza de las eximentes de la responsabilidad penal y demarcar con precisión los límites entre lo justificado y lo ilícito, lo legal y lo ilegal. Por su parte, el Catedrático de Derecho penal de la Universidad Pompeu Fabra, Jesús-María Silva Sánchez, en su artículo "Una crítica a las doctrinas penales de la lucha contra la impunidad y del derecho de la víctima al castigo" (pp. 35-56), desarrolla una sustantiva crítica aambas doctrinas penales, argumentando que tienen orígenes distintos y responden asimismo a motivaciones diversas, aun cuando cabe descubrir puntos de encuentro entre ellas, como el que se advierte cuando el derecho de lucha contra la impunidad se pretende justificar apelando a la satisfacción de un supuesto derecho de las víctimas a la Justicia (que se identifica con el castigo del autor). Elena Larrauri Pijuan, en "La economía política del castigo" (pp. 57-80), pasa revista al fenómeno de viraje de la filosofía de los sistemas punitivos desde una cultura penal asistencial a otra que enfatiza objetivos como castigo, incapacitación del delincuente y protección del público, que identifica con la cultura propia de los estados neo-liberales. En la segunda parte de su trabajo, analiza los factores protectores del punitivismo, focalizándose principalmente en tres: la economía política, la cultura política y el rol de los expertos, para terminar sosteniendo que una criminología avalada explícitamente en unos valores progresistas de solidaridad e inclusión de todas las personas y humana, que siga renegando del derecho penal para manejar los problemas derivados de la exclusión social, y fundada en la evidencia está en mejores condiciones para suministrar propuestas de políticas penales que permitan a nuestros representantes escapar a este círculo de desconfianza del público, gesticulación penal, casos de alarmante ineficacia, y una mayor desconfianza. La Catedrática en Derecho procesal de la Universidad de Girona, Teresa Armenta Deu, en un estudio comparativo entre Europa y América titulado "Exclusionary rule: convergencias y divergencias entre Europa y América" (pp. 81-110), da cuenta de las condiciones en que se ejerce la búsqueda de la verdad en el seno de un proceso a ambos lados del Atlántico, haciendo una pormenorizada referencia a los elementos subjetivo y objetivo de la regla de 


\section{BUSTOS, Reinaldo, "Recensión: Revista de Estudios de la Justicia, no 11 (2009)" Polit. crim. No 8, 2009, R1-8, pp. 7-9.}

exclusión, ilustrando la figura en diferentes aspectos singulares —actuaciones de sujetos privados; infiltrados; medios de prueba o carácter de las normas o derechos vulneradosque alcanzan especial valor en situaciones de incremento de la criminalidad organizada o más aún de amenaza terrorista. El artículo siguiente titulado "Medios alternativos de solución de conflictos y derecho penal" (pp. 111-130) de José Cid Moliné, de la Universidad Autónoma de Barcelona, en un trabajo de dos partes. Caracteriza en la primera parte la respuesta tradicional que ofrece el derecho penal frente al delito, para en la segunda parte refutar el paradigma proporcionalista y reseñar como alternativa, las alternativas a la prisión y la justicia restauradora; considerando que éstas concepciones alternativas proporcionan una respuesta más respetuosa a los intereses en juego y que, por ello, deberían plantearse, por lo menos, como la primera opción frente a la comisión de un delito. El Doctor en Derecho por la Universidad de Lérida, Luis Rodríguez Collao, en su artículo "Robo con homicidio" (pp. 131-152), asume la tarea de examinar desde una perspectiva crítica y dogmática la figura del robo con homicidio que contempla el artículo $433 \mathrm{n}^{\circ} 1$ de nuestro Código penal, subrayando los inconvenientes que trae consigo la reunión de dos conductas delictivas autónomas en un mismo tipo penal como estrategia de técnica legislativa, al mismo tiempo que analiza la estructura del tipo en sus aspectos objetivos y subjetivos, para efectuar algunas propuestas alternativas frente a las soluciones imperantes, cuya severidad - en su concepto - en muchos casos carece por completo de fundamento. El artículo "Culpabilidad y sujeto en la obra de Juan Bustos" (pp. 153-180), del profesor de Derecho penal de la Universidad Diego Portales, Jaime Couso, sintetiza las razones de la crisis de la concepción normativa de la culpabilidad, destacando específicamente los aportes de la concepción de Juan Bustos Ramírez para el debate contemporáneo sobre el fundamento material de esa categoría del delito, señalando también alguna de sus limitaciones. Según Couso, Bustos Ramírez pone tempranamente de relieve un aspecto fundamental para esta discusión, consistente en desplazar la condición de responsabilidad del sujeto individual a partir de criterios normativos a una concepción de la culpabilidad que requiere algo más que una atribución normativa, contenida en la idea de una exigibilidad social del cumplimiento de la norma, con la consecuencia de que, al asumir una dimensión histórica y política conflictiva de la atribución del papel del sujeto en el seno del orden social, se afirma una co-responsabilidad social por el conflicto criminal, que puede llegar a excluir la culpabilidad del sujeto, sin que por ello, éste pierda su condición de tal, que se deriva de su dignidad humana. Sin embargo, para el autor, la concepción de Bustos Ramírez tiene la limitación de que se hace cargo sólo de una dimensión de la autonomía que el concepto material de culpabilidad debería preservar. El artículo de Guillermo Oliver Calderón, Doctor en Derecho por la Universidad de Barcelona, "Seguridad Jurídica y derecho penal" (pp. 181-200), incursiona en el examen crítico del grado de cumplimiento que se observa en la práctica del derecho penal de las exigencias de las concepciones de la seguridad jurídica: la objetiva y la subjetiva: Así, en el apartado IV, examina la concepción objetiva, es decir, la que dice relación con la corrección estructural y funcional del sistema jurídico a través de sus normas e instituciones, las que entre otras cosas, implica que las leyes sean promulgadas y publicadas en forma clara y estricta, sin lagunas, irretroactivas y estables, y que los poderes públicos y los ciudadanos se sujeten al bloque de la ilegalidad, y; seguidamente, examina la concepción subjetiva que dice relación con la exigencia de que los ciudadanos sepan cuál es el derecho vigente para que puedan realizar conductas presentes y planifiquen sus futuras actuaciones con un grado razonable 
CENTRO DE ESTUDIOS DE DERECHO PENAL. "Revista de Revistas."

Polít. crim. No 8, 2009, R1-8, pp. 1-13.

[http://www.politicacriminal.cl/n_8/r_1_8.pdf]

de previsibilidad acerca de su valoración jurídica. Por su parte, el Profesor invitado de Derecho penal de la Universidad de Chile, Gonzalo Medina Schulz, contribuye con un extenso estudio titulado "Sobre la determinación de la pena y el recurso de nulidad en la Ley $N^{o} 20.084$ sobre responsabilidad penal adolescente" (pp. 201-234), haciendo especial hincapié en el examen de los criterios que en concreto dispone la ley para su consideración y valoración al momento de determinar la pena a imponer; de la posibilidad de revisión de las sentencias por cuestiones vinculadas al problema de determinación de las penas, y finalmente, sobre las causales del Recurso de Nulidad y sus relaciones con los aspectos relevantes del sistema de determinación de pena contemplada en la Ley de Responsabilidad Penal Adolescente. Finalmente, los aspectos doctrinarios se cierran con el artículo de Jonatan Valenzuela S., con estudios de Doctorado en la Universidad de Girona, titulado " $L a$ pena y la educación. Una aproximación al fundamento de la pena juvenil" (pp.235-264). El texto explora una teoría retributiva de la pena en el ámbito del derecho penal de menores, en contraposición a las justificaciones utilitarias.

Complementa este $\mathrm{n}^{\mathrm{o}} 11$ de la REJ, en la sección de Colaboraciones, los artículos de los abogados del Departamento de Estudios de la Defensoría Penal Pública, Ignacio Barrientos Pardo y Germán Echeverría Ramírez, quienes escriben respectivamente sobre el "Recurso efectivo contra la sentencia que no concede beneficios de la Ley $N^{o} 18.216$ " (pp. 265-318) $\mathrm{y}$, sobre la "Ultractividad en la persecución penal pública de las ofensas a la autoridad" (pp. 319-342); y del abogado Francisco Ferrada Culaciati, que escribe sobre "La constitucionalidad del artículo 149 inciso $2^{\circ}$ del Código procesal penal”' (pp. 343-370). 
MALDONADO, Francisco "Recensión: Anuario de Derecho Penal y Ciencias Penales, Vol. LXI (2008)" Polit. crim. No 8, 2009, R1-8, pp. 10-13.

\title{
Recensión: Anuario de Derecho Penal y Ciencias Penales Tomo LXI, fasc. único, Enero a Diciembre de 2008
}

\author{
Francisco Maldonado Fuentes \\ Doctor en Derecho. Profesor conferenciante Universidad de Talca y Director de extensión y \\ programas especiales del Centro de Estudios de Derecho Penal \\ fmaldonado@utalca.cl
}

El anuario trae en esta oportunidad ocho artículos de doctrina penal. Se inician con la contribución de Enrique Gimbernat Ordeig sobre el "Fin de la protección de la norma e imputación objetiva (pp. 5-30), seguidos por los artículos de Michael Pawlik sobre el "¿Engaño por medio del aprovechamiento de defectos de organización ajenos?: acerca de la distribución de riesgos conforme al 263 StGB en casos de errónea acreditación en cuenta y constelaciones emparentadas" (pp. 31-52); de Alicia Gil Gil acerca de "La autoría mediata por aparatos jerarquizados de poder en la jurisprudencia española" (pp. 53-88); de Miguel Angel Núñez Paz y, Germán Guillén López sobre "Entrega vigilada, agente encubierto y agente provocador: análisis de medios de investigación en materia de tráfico de drogas" (pp. 89-164); Gutmaro Gómez Bravo: "La política penitenciaria del franquismo en la consolidación del Nuevo Estado" (pp. 165-198); Félix María Pedreira González: "Derecho penal material, Derecho procesal penal y prohibición de retroactividad" (pp. 199-236); de Alvaro Mendo Estrella sobre "La compleja estructura de peligro en el denominado delito "ecológico" del artículo 325.1 del Código Penal: algunas alternativas" (pp. 237-264); y de Pedro-Alejo Llorente De Pedro sobre "La pena de presidio en las plazas menores africanas hasta la Constitución Española de 1812” (pp. 265-330). Se incluye además una Sección Legislativa a cargo de María del Carmen Figueroa Navarro (pp.331-378), una de jurisprudencia del Tribunal Constitucional, confiada a Gema Martínez Galindo (pp. 379-424); y una de Jurisprudencia del Tribunal Supremo español, que prepara María del Carmen Figueroa Navarro, Abel Téllez Aguilera (pp. 425-474). Además de la sección documental de la Fiscalía General del Estado (pp. 475-535) en la Sección de Bibliografía (pp.537-547) se reseñan los libros de Gonzalo Quintero O. sobre "La imagen de la Justicia en $\langle E l$ Quijote>, en Cervantes y en su tiempo"; de Juan Manuel Fernández Aparicio: "Derecho Penitenciario. Comentarios prácticos"; y de Francisco Bueno Arús: "Nociones de prevención del delito y tratamiento de la delincuencia", todos preparados por Carlos García Valdés. Remata con una nota acerca de los profesores que han accedido a la categoría de Catedráticos durante el año que abarca el Anuario.

Nos ha parecido adecuado extender nuestro comentario a propósito de la obra del prof. Pedreira González quien se adentra en las diferencias que plantea la aplicación de la prohibición de retroactividad en el derecho penal sustantivo y el procesal penal. Como es por todos conocido la perspectiva más tradicional asume que esta distinción entre lo material y lo adjetivo delimita el campo de aquello que quedará cubierto por la garantía en cuestión, centrándose por ello las dificultades a resolver en una adecuada adscripción de las diversas disposiciones en ambas categorías. Destaca que la discusión se ha visto cruzada e influenciada negativamente por la necesidad de resolver la aplicación de la prescripción respecto de los crímenes cometidos durante el nacionalsocialismo, discusión que se replica 
CENTRO DE ESTUDIOS DE DERECHO PENAL. "Revista de Revistas."

Polít. crim. No 8, 2009, R1-8, pp. 1-13.

[http://www.politicacriminal.cl/n_8/r_1_8.pdf]

en España a propósito de las pretensiones de Baltazar Garzón de avocarse al conocimiento de delitos cometidos durante la Guerra Civil (pp. 199-200). Dedica el cuerpo del artículo a pasar revista a las teorías más relevantes de considerar para rematar en el análisis de la llamada teoría de la supresión mental del proceso que plantea Hilde Kaufmann en el año 1968, en base a la tradicional distinción entre acción y pretensión de Windscheid, destacando que se trata de una de las aproximaciones más completas sobre el particular. No se inclina sin embargo por dicha tesis, asumiendo, según veremos, una construcción mas bien clásica radicada en Binding. Describe inicialmente la llamadas teoría negativa (J. Binder) como una forma de comprensión que niega la existencia de una distinción relevante entre los aspectos materiales y los procedimentales de un derecho, asumido como una relación jurídica que forma parte de los ámbitos ideales y que sólo es <imaginada $>$ por el juez en cuanto recreada en el proceso a través de las pruebas. Se trata de una construcción que presenta como falencia el que a fin de cuentas reconoce una pre-existencia de lo material, lo que formalmente es negado como supuesto, planteando una contradicción difícil de resolver o compatibilizar. Hay, en efecto, una afectación del derecho que es previa a la interacción (pp. 214-5) procesal y que tiene existencia propia y relevancia para el proceso (p. 204). Asimismo se le atribuye inevitablemente un valor constitutivo a la sentencia, elevando a los jueces a la categoría de creadores del derecho. Ejemplifica, convincentemente, en base a la presunción de inocencia y la pretensión que nace de la comisión de un delito (p. 205). Por lo demás no se explica la presencia de derechos satisfechos sin que exista un proceso de por medio (p. 206), cuestión que si bien resulta ajena al derecho penal, resulta determinante en el rechazo de la propuesta en global. En la misma línea cita la tesis de H. Mayer acreedora de idénticas observaciones constitutivas ( $\mathrm{p}$. 206). La necesidad de acudir al proceso ( máxime en materia penal ) no lleva en modo alguno a anular las distinciones presentes entre lo material y lo adjetivo, ni la autonomía de las disciplinas que lo abordan. Finalmente, destaca que esta misma línea reaparece recientemente (Volk) de la mano de la constatación de equivalentes funcionales pertenecientes a ambas ramas (pp. 207 y ss.), tesis que se rechaza en base a desacreditar que el derecho penal y el procesal penal tengan los mismos fines (aseguramiento de la paz jurídica y la confirmación del derecho). Destaca la presencia de discursos similares en planteamientos de Octavio García Pérez, Bustos Ramírez, Hormazábal Malarée y Gili Pascual, particularmente a los efectos de la construcción de excusas absolutorias en determinados ilícitos de carácter patrimonial a partir del principio de insignificancia (pp. 209-212). En cuanto a los denominados criterios formales, destaca inicialmente la teoría de la conexión con el hecho, introducida por Gallas en la Gran Comisión, sosteniendo que la válvula radica en el contenido de garantía del principio de legalidad. Sobre esa base sólo lo que forma parte de las bases de la punibilidad, o sea, lo que está conectado con el hecho, será derecho material (sistematizado como condición objetiva de punibilidad). Asocia esta tesis con la pretensión de prolongación retroactiva de los plazos de prescripción (p. 213). Destaca la adhesión de Schmidthäuser, Stratenwerth y posteriormente de Roxin, abordando el desarrollo particular que proponen estos últimos autores (pp. 214-216). Lo central es que se asume que no puede depender la punibilidad (material) de un elemento ajeno al hecho, de lo que dependerá a fin de cuentas la pertenencia al ámbito de lo adjetivo y de lo procedimental. Entre las críticas se cuentan que no permite explicar que se reviva la punibilidad ya extinguida, por ej. en caso que la prescripción haya vencido (p. 216), sobre 
todo a partir de las diferentes bases que llevan a sustentar materialmente el principio de legalidad. Así no es claro si este se refiere a todo el derecho penal material o solo a aquello que se orienta por el principio de confianza, llegándose en ambos casos a ámbitos de inclusión (en el derecho material) considerablemente diversos. De esta forma la segmentación depende más bien y autónomamente del alcance del principio de irretroactividad que de la conexión con el hecho (pp. 217-221). Lo propio acontece con quienes asocian la relación factual con la idea de necesidad de pena, objeciones desarrolladas de la mano de H. Kaufmann (pp. 221-222). Finalmente se señala que el costo marginal de la tesis es cuestionar que los presupuestos de punibilidad estén efectivamente fuera del hecho y que se obvia por completo la consideración de los fines del proceso en todas las distinciones propuestas (p. 222). Aborda finalmente las teorías que recurren a criterios materiales. Inicialmente expone la llamada teoría de las normas primarias y secundarias de Nawiasky, asociadas, respectivamente, al derecho material y procesal (pp. 223-227), según si prescriben o no un comportamiento (las demás, se dirigen al órgano resolutor). Se le objeta la identificación del derecho material con la relación de deber instaurada en la norma y la confusión que plantea entre los aspectos formales y materiales de la misma (p.224), destacados ya en su trascendencia por la distinción planteada en similares términos por Binding. Además, no permite reconocer normas previas a la formulación de la tipología penal ni reconocer imperativos independientes de la sanción, ni menos aun el que los ciudadanos son destinatarios de las prescripciones penales. Se dedica a continuación a la teoría del merecimiento de pena atribuida a Bemmann, a partir de los conceptos (originarios) de Beling. La base es que siempre en las normas penales se trata de conocer si debe imponerse una pena, cual debe ser ésta y bajo qué circunstancias, mientras que la normativa procesal busca determinar si se debe proceder, cómo (ritualidad) y bajo qué requisitos. De esta forma lo penal dice relación con el merecimiento de pena mientras que lo procesal con la actividad persecutoria (p. 228). Se le objeta la dificultad de llevar a cabo dicha distinción en concreto, esto es, a la hora de enfrentarse a un caso de difícil solución, el que restringe excesivamente el ámbito del derecho material, que sólo resulta practicable en una perspectiva retributiva, objeciones particularmente relevantes en la tesis concreta de Bemmann y agregándose, en el caso de Binding, la generalidad de su propuesta. Pedreira González plantea sin embargo una visión más bien favorable a esta formulación, asumiendo que los contenidos que sirven de base a la crítica dicen más bien relación con las particulares acotaciones de los autores que la defienden más que con la propuesta en sí misma. Aborda asimismo contradicciones similares presentes en teorías alternativas, procurando sobre esa base fortalecer su punto de vista (p. 231). Finalmente se hace cargo de la tesis de Kaufmann basada en lo que titula como la supresión mental del proceso. Para la autora alemana la cuestión depende de que no pensemos en el proceso, solución ideada para el derecho civil y que propone trasladar al ámbito del derecho penal, al menos hipotéticamente (asumiendo la imposibilidad de imponer una pena sin proceso). Se trata de ver si la norma cuestionada en su naturaleza es o no irrelevante para determinar la imposición de una pena, asumiendo que una respuesta afirmativa lleva a adscribirla al derecho procesal (p. 232). Se le objeta su carácter hipotético, la falta de contenido, el que no permite la solución en caso de los llamados elementos mixtos. Como ya anticipamos, Pedreira adscribe a la tesis de Binding, asumiendo que proporciona una adecuada pauta material de solución, que debe ser complementada con una nota aclaratoria también 
CENTRO DE ESTUDIOS DE DERECHO PENAL. "Revista de Revistas."

Polit. crim. No 8, 2009, R1-8, pp. 1-13.

[http://www.politicacriminal.cl/n_8/r_1_8.pdf]

aplicable a dicho autor, cual es, que los presupuestos de merecimiento de pena se extienden mas allá de la culpabilidad (p. 236). 